\title{
Biological Clocks and the Coordination Theory of RNA Operons and Regulons
}

\author{
J.D. KEENE \\ Department of Molecular Genetics and Microbiology, Duke University \\ Medical Center, Durham, North Carolina 27710
}

\begin{abstract}
One of the regulatory models of circadian rhythms involves the oscillation of transcription and translation. Although transcription factors have been widely examined during circadian processes, posttranscriptional mechanisms are less well-studied. Several laboratories have used microarrays to detect changes in mRNA expression throughout the circadian cycle and have found that mRNAs encoding the RNA-binding proteins (RBPs) nocturnin and butyrate response factor (BRF1) undergo rhythmic changes. Nocturnin is a deadenylation enzyme that removes poly(A) from the 3' ends of mRNAs, whereas BRF1 destabilizes mRNAs encoding early response gene (ERG) transcripts that contain AU-rich sequences in their $3^{\prime}$-untranslated regions (UTRs). Moroni and coworkers proposed that BRF1 functions as an oscillating posttranscriptional RNA operon (PTRO) that diurnally degrades ERG transcripts in peripheral organs (Keene and Tenenbaum 2002; Benjamin et al. 2006). The PTRO model posits that mRNAs can be members of one or more discrete functionally related subsets of mRNAs as determined by cis elements in mRNA and trans-acting RBPs or microRNAs that collectively recognize these cis elements (Keene 2007). This chapter describes the basis of posttranscriptional coordination by RNA operons and their potential for horizontal transfer among cells and discusses the potential for RBPs and microRNAs to participate in coordinating circadian rhythms and other biological clocks.
\end{abstract}

\section{INTRODUCTION}

In bacterial and eukaryotic cells, gene expression is regulated at both the transcriptional and translational levels. However, in bacteria, unlike that of eukaryotes, the transcription apparatus is directly coupled to the translation apparatus. Thus, bacterial transcripts begin translation even before transcription is completed because the ribosomes initiate translation on nascent transcripts (Beckwith 1996). Eukaryotic transcription and translation cannot be directly coupled because the nuclear membrane separates the chromosomes from the ribosomes requiring RNA processing and export between transcription and translation (Keene 2001; Maniatis and Reed 2002). Recent findings suggest that the steps between transcription and translation in eukaryotes are interconnected and that each step in the pathway is physically connected to the next (Fig. 1). Thus, as transcripts are transferred from the DNA template to the spliceosome, the splicing apparatus itself is coupled to the nuclear export machinery, and following export, mature transcripts are transported to cytoplasmic locations where their final fates are determined (for review, see Maniatis and Reed 2002). mRNAs contain sequence elements that help determine their fates both during and after export to the cytoplasm (Richter 1997; Keene 1999, 2001, 2003). Therefore, although the molecular interconnectivity of the steps between transcription and translation in eukaryotes provides indirect coupling of RNA-processing events, each mRNA and its bound proteins is assumed to function as a free agent. But is there coordination of groups of mRNAs in parallel along this pathway? The PTRO theory is based on evidence suggesting that the pathway of RNA processing is indeed coordinated and has implications for mechanisms of biological timing such as those involved in circadian rhythms and other biological clocks (Keene and Tenenbaum 2002; Keene 2007).

\section{COUPLING AND COORDINATION OF GENE EXPRESSION FROM TRANSCRIPTION TO TRANSLATION}

Control of gene expression at the level of transcription in mammalian cells appears to be regulated by transcription factors that bind specifically to multiple promoter elements (Orphanides and Reinberg 2002; Kosak and Groudine 2004). It is assumed that gene expression is coordinated by this mechanism, but there is not abundant experimental evidence to directly support this assumption. Models of transcriptional regulation state that multiple genes are activated as functional groups because they use common promoters, but the activation of transcription appears to occur in domains or neighborhoods where chromatin is modified to allow access to RNA polymerase and the transcription apparatus. For example, "ectopic expression" of genes that are physically located near one another in neighborhoods results from the decondensation of chromatin that makes genes accessible to activation (Rodriguez-Trelles et al. 2005; Aten and Kanaar 2006). Several studies have demonstrated that these ectopically expressed genes do not necessarily have related functions, and thus, the physical proximity of coexpressed genes does not correspond to the functional relatedness of those genes (Spellman and Rubin 2002; Lercher et al. 2004; Yanai et al. 2006). Thus, ectopically coexpressed genes are by definition temporally coordinated, but this has been interpreted to result as much from "leakage" of transcription as it does from mechanistic coregulation (Rodriguez-Trelles et al. 2005; Yanai et al. 


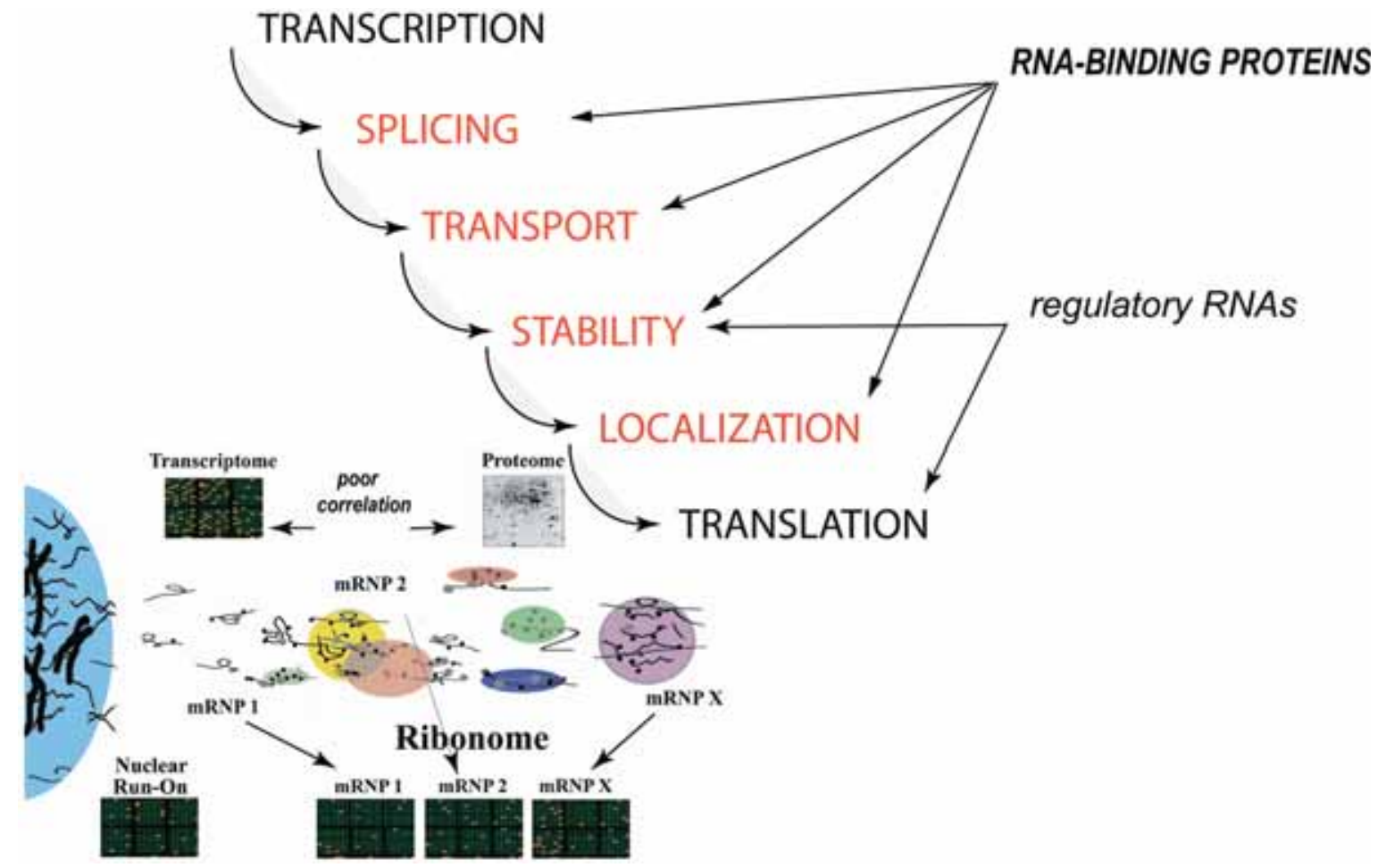

Figure 1. Levels of mRNA processing that are responsible for the interconnectivity of transcription to translation in eukaryotic cells that are regulated by RBPs and small regulatory RNAs such as microRNAs (Keene 2001, 2007). These processes are interconnected and coordinated by a dynamic RNP-driven process of regulation of multiple mRNAs in parallel. (Inset) Ribonome (the cellular ribonucleoprotein infrastructure) and the RIP-Chip approach of using microarray analysis of enriched RNPs and their associated mRNA subsets. A full analysis of the structural and functional relationships among mRNAs identified using RIP-Chip together with other informatics methods is termed ribonomic analysis (Tenenbaum et al. 2000, 2002, 2003).

2006). But the coordination of expression of functionally related groups of human genes is assumed to orchestrate complex biochemical pathways and developmental events such as "synexpression" (Niehrs and Pollet 1999). In fact, it is not known precisely how gene expression is coordinated in mammalian and other eukaryotic cells, but it is likely important for the proper performance of all cellular and developmental processes as well biological clocks.

\section{REGULATION OF GENE EXPRESSION BY RNA-BINDING PROTEINS AND MICRORNAS}

Although the assumption of a transcriptional basis for gene coordination in eukaryotes is widely assumed by most biologists, it has recently been discovered that posttranscriptional events as well can be coordinated (Keene 2007). Moreover, coordinated posttranscriptional regulation is important because these late events in the gene expression pathway dictate the final decisions of protein production regardless of how precisely transcription itself is coordinated (Fig. 1) (Keene 2001, 2007; Keene and Tenenbaum 2002). Indeed, evidence suggests that RBPs are multitargeted (Gao et al. 1994; Tenenbaum et al. 2000; for review, see Keene 2007) and such targeting has also been proposed more recently for microRNAs and possibly other small noncoding RNAs (Lewis et al. 2003, 2005; Bartel 2004).

\section{MULTITARGETING OF MRNAS BY RBPS}

One of the key requirements of coordinated gene expression is that the mRNA transcripts that express proteins are capable of being coregulated. For example, Gao et al. (1994) provided early evidence that RBPs may be targeted to multiple brain mRNAs in vitro. This idea of multitargeting was unique in that the mRNAs found to interact with ELAV/Hu proteins were believed to have related functions and potentially to respond together in neuronal or immune cells as ERG transcripts (Keene 1999; Brennan and Steitz 2001). These mRNAs were known for many years to contain a characteristic set of sequence elements that were AU-rich (ARE), and these genes encoded such proteins as c-Myc, c-Fos, interleukins, interferons, and many otherwise "dangerous" proteins that mammalian cells presumably need to keep under tight control (Shaw and Kamen 1986). A signature sequence of the ARE was the pentameric AUUUA or the larger but less-frequent seven-nucleotide consensus sequence UAUUUAU, but these elements were essentially U-rich and scattered with A and G residues (Levine et al. 1993; Gao et al. 1994). Most importantly, these regions of sequence similarity were never identical among these mRNAs but again represented a sequence of similar character. The lack of sequence identity made it exceedingly difficult to globally identify such elements among mRNAs using computational methods (Keene 2001). In essence, these early 
experiments were designed to allow the ELAV/Hu RBPs, rather than the computer, to inform us of their targeting specificity for multiple mRNAs. Thus, the demonstration by Gao et al. (1994) that the ELAV/HuB protein could bind to these $\mathrm{A} / \mathrm{G}-\mathrm{U}$-rich sequences en masse led to the suggestion that functional consequences of such binding may coordinate functional outcomes by the ELAV/HuB RBPs in neural tissues. However, in vitro approaches do not address the key question of intracellular context of the protein and the RNAs with which it may be able to interact, and this rationale represents an ongoing experimental and technical effort in our laboratory and the field to develop methods that reveal these interactions in a functional cellular context.

The discovery and functional studies of the human ELAV/Hu RBP family followed from studies in Drosophila where Kalpana White and coworkers discovered the elav locus and demonstrated that the ELAV proteins were essential for neuronal differentiation in flies (Robinow et al. 1988). Complementary DNAs encoding four human neuronal ELAV/Hu proteins (HuA [HuR], $\mathrm{HuB}, \mathrm{HuC}$, and $\mathrm{HuD}$ ) were subsequently cloned in the Furneaux and Keene laboratories (Szabo et al. 1991; Levine et al. 1993; King et al. 1994; Ma et al. 1996). As noted above, the RNA-binding specificity of ELAV/Hu family proteins was demonstrated for ARE-type sequences found in the proto-oncogene and cytokine ERG mRNAs (Levine et al. 1993; Gao et al. 1994). HuB protein was found to have a binding preference for short stretches of uridylates flanked by A or G, and sometimes even C residues (Levine et al. 1993; Gao et al. 1994). In addition, $\mathrm{HuB}$ was found to stabilize an endogenous ARE-containing target mRNA that encodes the glucose transporter 1 protein (GLUT1) in adipocytes and, at the same time, to increase its translation by 20-50-fold (Jain et al. 1997). Subsequently, all four of the Hu family proteins, including the ubiquitously expressed $\mathrm{HuR}$ protein (also called $\mathrm{HuA}$ and ELAVl-1), were found to bind similar ARE sequence elements and to stabilize reporter mRNAs containing AREs (for review, see Keene 1999; Brennan and Steitz 2001). In addition to the HuB activation of GLUT1 mRNA translation, $\mathrm{HuB}$ translational activation of neurofilament M (Antic et al. 1999), HuR translational activation of $\mathrm{p} 53$ (Mazan-Mamczarz et al. 2003), and HuR translational activation of the SIRT1 mRNA (Abdelmohsen et al. 2007) were subsequently demonstrated in the absence of effects on RNA stability. In many other cases, effects on RNA stability with subsequent effects on translation were observed (for review, see Brennan and Steitz 2001; Keene 2001).

\section{MULTITARGETING OF MRNAs by MICRORNAS}

MicroRNAs have also been suggested to act through a multitargeting mode (Lewis et al. 2003, 2005). The data in support of multitargeting by microRNAs were initially based on computational predictions using algorithms such as TargetScan, PicTar, and MirAnda, each of which has its own advantages and disadvantages. In general, however, all of these methods examine microRNAs and potential mRNA targets in large databases for sequence complementarity. The basis of the homology search is the conservation of microRNA-targeted mRNA sequences of approximately six to eight nucleotides that potentially bind to "seed" sequences (Lewis et al. 2003, 2005). This very small region of homology is restricted by searching databases for the conservation of the mRNA complementary sequence among species, thus improving the potential of the sequence match having biological significance. More recently, these methods were refined to consider other determinants of microRNA function on 3'UTR sequence elements and to provide a prediction model that quantitatively evaluates the context of the microRNAmRNA interactions (Grimson et al. 2007). Subsequent attempts to directly demonstrate multitargeting have met with some degree of success, but issues regarding RNA structural constraints, "off-target effects," and cellular context remain to be understood. Thus, whether one is concerned with RBPs or microRNAs, it is essential to understand the in vivo intracellular context of the RNA-protein and RNA-RNA interactions. For this purpose, we devised methods to isolate ribonucleoprotein (RNP) complexes from cell extracts and to identify proteins and RNAs that associate with them (Fig. 2) (Keene et al. 2006).

Discrete subpopulations of mRNAs reside in RNP complexes and can be coordinately processed: spliced, transported, stabilized or degraded, localized, or translated into protein. All posttranscriptional steps involve the proper functioning of RBPs as members of these RNP complexes (see mRNP1, mRNP2, mRNPx in Fig. 1 inset). Our laboratory devised a method termed "RIPChip" or "RIP-on-Chip" (RNP-ImmunoprecipitationmicroChip) and found that RBPs can interact with distinct subsets of the mRNAs that together encode functionally related proteins (Fig. 2) (Tenenbaum et al. 2000; for review, see Hieronymus and Silver 2004; Keene and

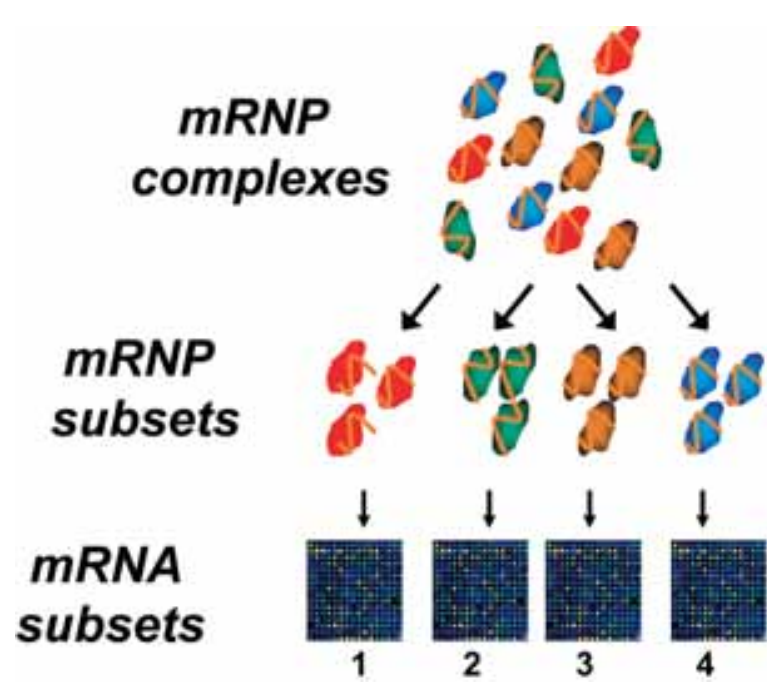

Figure 2. Depiction of the RIP-Chip concept in which mixtures of mRNPs are isolated from cell extracts and partitioned using antibodies or epitope tags that bind to proteins in the mRNP complexes. RNPs are released and the extracted RNAs are identified using microarrays or high-throughput sequencing. Small noncoding RNAs such as microRNAs that are associated with RNPs can be identified also using RIP-Chip (Keene et al. 2006). 
Lager 2005; Keene et al. 2006). Data from many laboratories indicate that RBPs in yeast, flies, and mammals can function together to form gene expression networks regulating multiple functionally related mRNAs (for review, see Keene and Tenenbaum 2002; Hieronymus and Silver 2004; Keene and Lager 2005; Moore 2005; Keene 2007). Similar approaches in our laboratory also have indicated that specific subpopulations of microRNAs can be identified in association with the ELAV/HuR protein in human Jurkat cells (P.J. Lager and J.D. Keene, unpubl.).

\section{RIBONOMIC ANALYSIS AND \\ RIP-CHIP PROVIDE INSIGHTS INTO MULTITARGETING OF MRNAS}

RIP-Chip is useful for reducing the complexity of transcriptomic data and at the same time allowing the simultaneous assessment of posttranscriptional regulation of multiple genes with the goal of better understanding the intracellular organization of mRNA transcripts (Figs. 1 and 2) (Tenenbaum et al. 2000, 2002). This has been called "ribonomics," because it allows one to determine the natural clustering of transcripts by RBPs en masse. The ribonomic approach is based on the use of RIP-Chip for purification of RNP complexes, followed by the identification of the mRNAs that are associated with the complexes on a microarray or by direct sequencing. The ribonomic analytical methods developed by Tenenbaum et al. (2000) consist of four major steps that are intended to derive relevant ribonomic information from RIP-Chip data. The association of proteins, mRNAs, and microRNAs with multiple mRNP complexes forms the "ribonome," and as described below, this kind of analysis provides functional information regarding the mRNA population of the cell (Fig. 3) (Brown et al. 2001; Eystathioy et al. 2002; Keene and Tenenbaum 2002; Hieronymus and Silver 2003).

The first step in the general ribonomic procedure is to isolate mRNPs using specific molecular tags, antibody epitopes, or other ligands that are associated with the mRNP complex using the RIP-Chip procedure (Fig. 2) (Tenenbaum et al. 2002, 2003; Keene et al. 2006). The conditions of the isolation can be optimized biochemically so that peripheral components of the mRNP complex are removed while leaving the RBP still associated with the mRNA targets in the RNP complex. If necessary, the RBP and its RNA targets can be cross-linked using a chemical such as formaldehyde or UV light, but this can introduce other complicating issues that are not addressed here for lack of space (see Penalva et al. 2004; Keene et al. 2006). In the second step of the general ribonomics procedure, the mRNA(s) in the isolated mRNP complex is identified. There are several methods available to identify the mRNAs including (1) the generation and sequencing of cDNA libraries from the isolated mRNAs, (2) the generation and sequencing of reverse transcription-polymerase chain reaction (RT-PCR) products, (3) specific probe RNase protection assays, or (4) microarray analysis. High-throughput sequencing procedures such as the "4-5-4" sequencing procedure that is a form of "mas-

\section{BACTERIAL POLYCISTRONIC MRNA}

\begin{tabular}{|l|l|l|l} 
ORF 1 & ORF 2 & ORF 3 & ORF 4 \\
\hline
\end{tabular}

\section{EUKARYOTIC MONOCISTRONIC MRNAS}

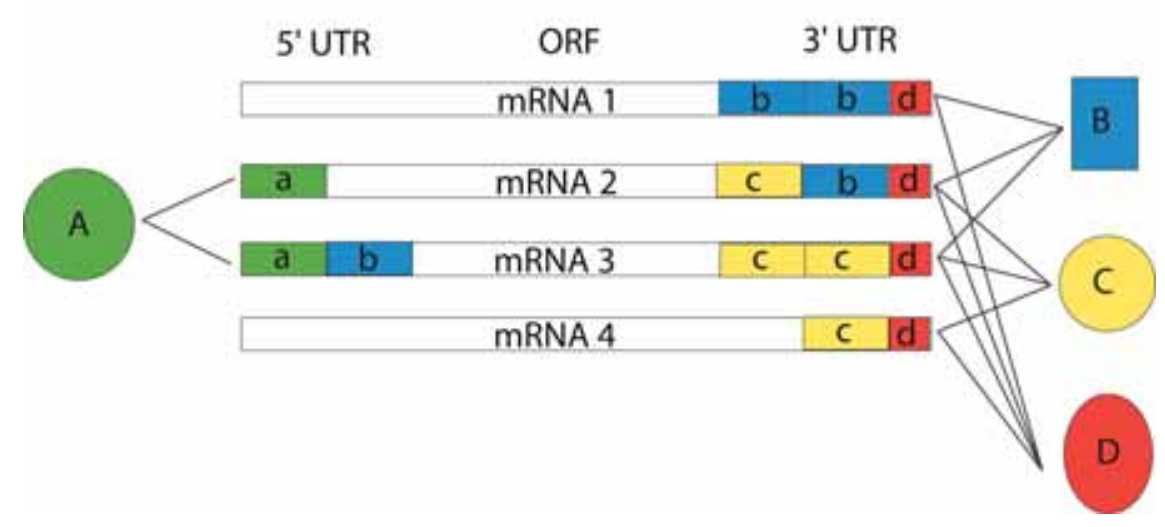

Figure 3. Model depicting the PTRO theory. (A) An analogy to bacterial polycistronic DNA operons and their collinear mRNAs is suggested, and it was proposed that in eukaryotes $(B)$ monocistronic mRNAs are regulated by multiple RBPs and microRNAs to activate or repress distinct groups of mRNAs. The model accounts for multilevel regulation of functionally related groups of mRNAs in different combinations by trans-acting factors. This allows each mRNA to be a member of more than one RNA operon so that the encoded proteins can be produced coordinately in different combinations via distinct UTR codes (Keene and Tenenbaum 2002). (Reprinted, with permission, from Keene and Tenenbaum 2002 [C Elsevier].) 


\section{RNA OPERONS, REGULONS, AND BIOLOGICAL CLOCKS}

sively parallel sequential sequencing" (MPSS) or "singlemolecule platform sequencing" (SMPS) can be used directly to provide complete and unbiased sequence evaluation of RIP-Chip-derived RNAs.

The third and fourth steps of the general ribonomics procedure involve determining the biological significance of the experimental outcome. These steps are more complex and require solving problems that can be technically challenging. If there are multiple targets observed in step two, the third step is used to determine which features that the RNAs found in the RNP subpopulation have in common. For example, is there a recognizable binding site for the RBP of interest (e.g., an ARE-type)? As described above, functionally similar mRNAs may have similar but not identical sequences in common for binding to RBPs, and these sequences are likely to be semiconserved among mRNAs in order for them to interact with the same trans-acting RBP. Tenenbaum et al. (2000) found that mRNAs identified by microarrays following immunoprecipitation of the ELAV/HuB protein contained ARE sequences of similar character that resemble known consensus RNA-binding sequences for ELAV/Hu proteins. This consensus was confirmed but further described within the context of a secondary structure by Lopez de Silanes et al. (2004) using RIP-Chip. Similar findings involving both primary and secondary mRNA structures were reported for the Fragile-X mental retardation protein by Brown et al. (2001) and for mRNAs bound to nuclear export proteins in yeast by Hieronymus and Silver (2003). Two of the most striking recent examples of subsets of mRNAs that associate with Pumilio RBPs are those that encode macromolecular structures such as the mitochondrion (Gerber et al. 2004) or the vacuolar proton-translocating V-type ATPase complex (Gerber et al. 2006), as well as multiple components that regulate anterioposterior patterning of Drosophila embryos (Gerber et al. 2006). Many other examples have been summarized by Keene and Lager (2005; Keene 2007). More recently, RIP-Chip was used with the histone mRNA stem-loop binding protein (SLBP) and demonstrated to interact with approximately 30 histone mRNAs with high specificity (Townley-Tilson et al. 2006). Five of the key histones involved in cell-cycle-specific coordination of chromatin reformation during $\mathrm{S}$ phase were temporarily associated in a manner that suggests a distinct PTRO (Fig. 4).

The fourth step is the most difficult of all because it is concerned with the functional relationships among the proteins encoded by the mRNAs that are identified. This is the most biologically important information to be obtained from the procedure and it may lead to a useful systems biological outcome. The best approach is to use various gene ontogeny $(\mathrm{GO})$ databases such as Panther to search for

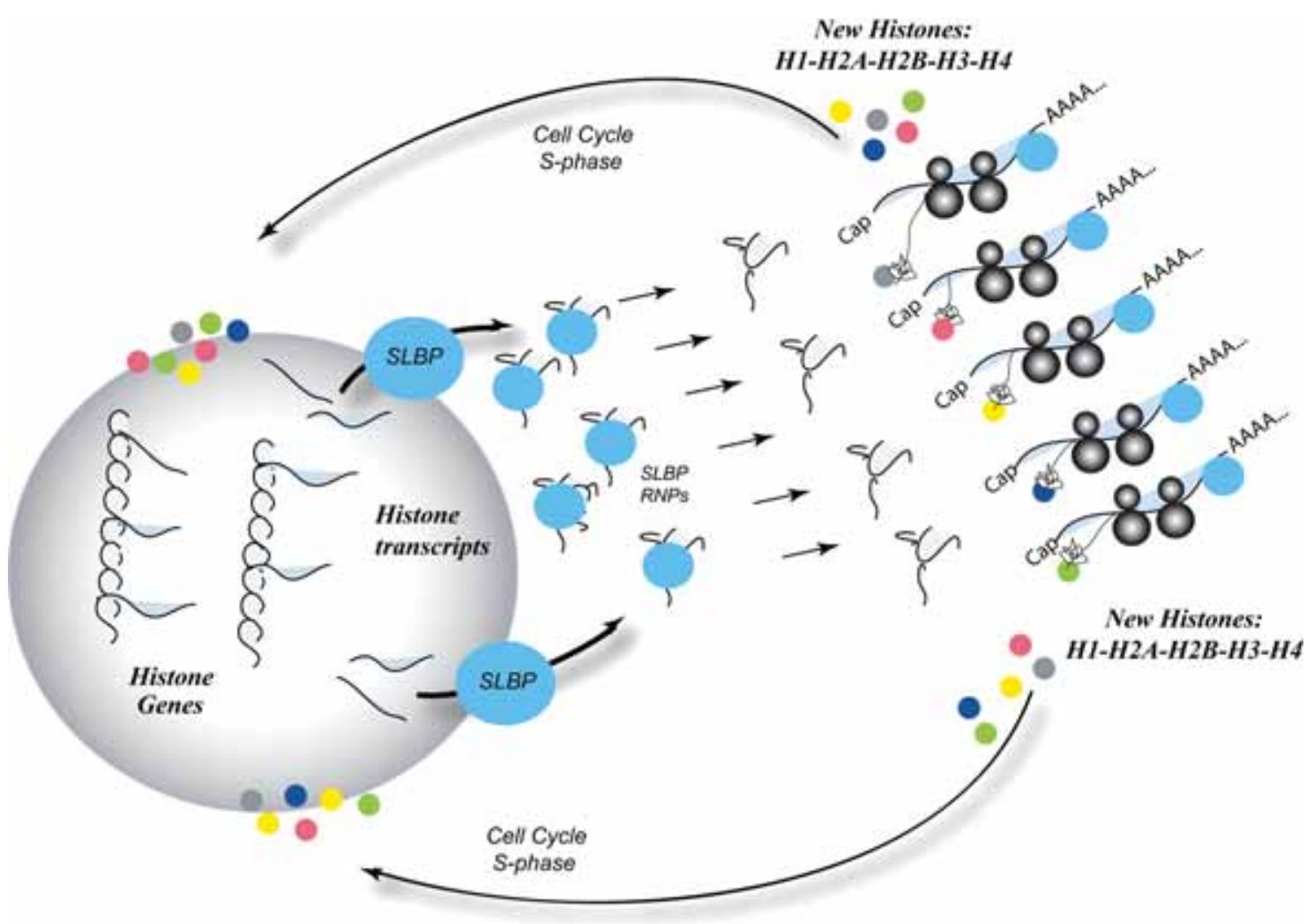

Figure 4. Depiction of the coordinated production of replication-dependent histone proteins H1-H2A-H2B and H4 encoded by a subset of mRNAs that are coregulated by the mammalian stem-loop-binding protein (SLBP) (Townley-Tilson et al. 2006). The histone genes are dispersed across the genome, but the SLBP binds to a 3'UTR element in the histone transcripts and coordinates the translation of the histone mRNAs. The mRNA subset that is bound to SLBP functions as a PTRO for temporal coordination of histones together with DNA synthesis and chromatin assembly during the cell cycle. 
functional relationships and to then expand the analysis by linking multiple functional groupings together to visualize a potential RNA operon or higher-order regulon (Fig. 3) (Keene and Tenenbaum 2002; Keene 2007). The principle of a ribonomics analysis is that the association of a class of mRNAs with an RNP complex or an RBP serves as an indication that the mRNAs function together as a group. Cells may use this natural clustering of mRNAs to regulate the production of proteins that function together in a pathway or coordinate the synthesis of a biological machine such as a ribosome or spliceosome.

It should also be noted that other methods and experimental approaches have been used that have led to conclusions similar to those obtained using RIP-Chip (for review, see Keene and Lager 2005; Keene 2007). These include polysome gradient shifts, en masse microarray analysis of mRNA decay, and genetic methods in yeast and flies. Thus, in aggregate, the posttranscriptional PTRO model as depicted in Figure 3 and described below has been strongly supported by these various types of experimental data.

\section{COORDINATED GENE EXPRESSION AND THE POSTTRANSCRIPTIONAL OPERON MODEL}

The theory of the PTRO proposes that mammalian cells organize monocistronic mRNAs in functional groups much like polycistronic mRNAs cluster functionally related gene products in bacteria (Fig. 3) (Keene and Tenenbaum 2002). As noted above, in bacteria, the polycistronic DNA operons are highly efficient at coordinating the expression of functionally related proteins, but the mechanism is constrained by the inability of each protein to be expressed independently or as part of another functionally related subpopulation. Furthermore, these monocistronic mRNAs can be regulated independently of one another or in groups by trans-acting RBPs or microRNAs (represented by A, B, C, D) that bind cis-acting sequences (represented by a, b, c, d) in their 3'UTR and 5'UTR. This model provides regulatory independence of expression for proteins encoded by the monocistronic mRNAs to adopt new functions and to regulate the production of each functional version of the protein at distinct intracellular locations or under different temporal modes either alone or together with proteins of related function (Keene and Tenenbaum 2002). Thus, the independently functioning forms of each protein can be regulated posttranscriptionally via separate $c i s$-acting elements in the mRNA. The power of this architecture for higher cells is that mRNA members of mRNA subpopulations can be reassembled in different combinations depending on the regulatory signals and growth state of the cell (Hieronymus and Silver 2004; Moore 2005; Keene 2007). Therefore, one can imagine how a modest number of human genes (e.g. <30,000) can be used in multiple combinations to organize complex functions required for the development of a multicellular organism. In sum, PTROs provide a mechanism to coordinate the final outcome of gene expression in eukaryotic cells (for review, see Hieronymus and Silver 2004; Wilusz and Wilusz 2004; Fan et al. 2005; Keene and Lager 2005; Moore 2005; Vemuri and Aristido 2005; Keene 2007).

\section{POTENTIAL FOR HORIZONTAL TRANSFER OF PTROS}

We have extended the PTRO theory based on recent evidence to include the potential for the horizontal transfer of PTROs (Keene 2007). This corollary to the PTRO theory proposes that exosomes and microvesicles contain PTROs, and the associated mRNAs and microRNAs are exchanged among these cells by exosome-mediated transfer (Fig. 5) (Valadi et al. 2007). More recently, Aliotta et al. (2007) demonstrated that RNAs contained in exosomes from radiation-treated lung cells could transfer a phenotype to bone marrow cells. In addition, conditioned media and secreted microvesicles derived from other cell types have been shown to alter the phenotypes of recipient cells. For example, embryonic stem cells were shown to produce RNA-containing microvesicles that could reprogram hematopoietic progenitor cells (Ratajczak et al. 2006). Similar claims of phenotypic transfer of mRNAs by microvesicles and exosomes from tumor cells to monocytes have been described recently (Baj-Krzyworzeka et al. 2006). This has exceedingly important implications for all biological systems. We theorize that specific mRNAs and microRNAs representing PTROs are involved in cell-cell communication during growth, differentiation, and environmental assault, including cellular repair and cell death, and potentially circadian rhythms. These processes take place within the cellular infrastructure of the ribonome (Figs. 1 and 5) (Keene 2001).

\section{THE RIBONOME AND POSTTRANSCRIPTIONAL REGULATORY NETWORKS}

The ribonome consists of thousands of RBPs and their associated mRNAs that encode those RBPs and other proteins. Each RBP-associated mRNA is capable of being regulated by other RBPs within the ribonome that can bind to it and determine its fate. Thus, each RBP's own mRNA can be translationally activated to replace that RBP only when the community of bound RBPs allows it (see reciprocal regulatory interactions between ribonome and proteome in Fig. 5). Thus, within the ribonome are regulators-of-regulators (i.e., RBPs acting on one another's mRNAs) to keep the environment in balance. Although some RBPs activate translation of mRNAs, other RBPs inhibit translation, thus providing safeguards to the network of overlapping feedback loops, feed-forward loops, and commands for self-replenishment of the ribonome to maintain a balanced yet dynamic entity. Although the ribonome is self-sustaining, it is also selflimiting given these regulatory loops, and therein resides its central functional role as a management site for cell growth and multicellular expansion (Fig. 5).

The concept of the ribonome as a central managerial entity in biological systems is consistent with both a closed and an open system because it allows its own infrastructure to be self-regulating while regulating multiple non-RBP mRNAs (Figs. 1 and 5). Thus, each of the RBPs in the system can regulate one another's mRNAs while also regulating multiple other mRNAs that do not 


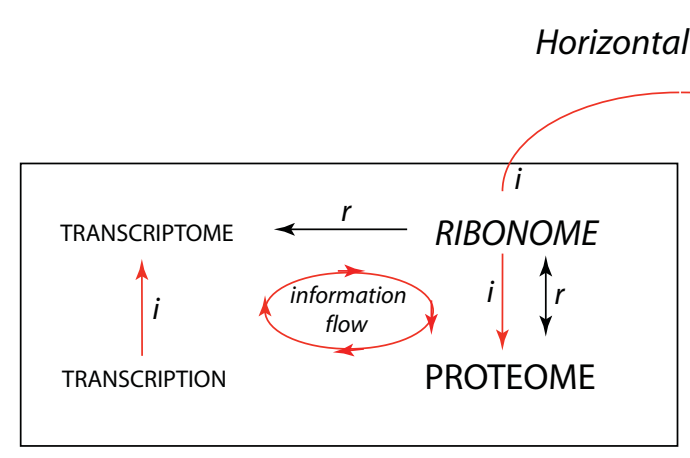

Cell \#1

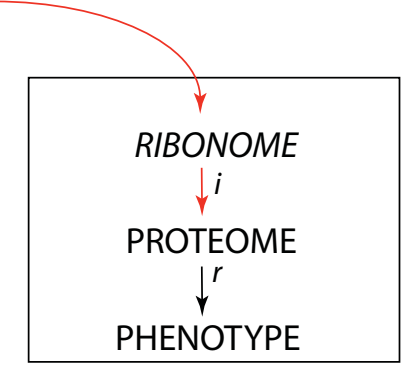

Cell \#2

Figure 5. Depiction of a proposed mechanism of intercellular horizontal transfer of RNA. Cell \#1 shows the interdependence of the ribonome and the proteome in both information transfer $(i)$ and regulatory $(r)$ modes. Information flows routinely from transcription to the proteome (central circle). Transcription feeds the transcriptome that is in turn shaped by trans-acting factors (e.g., RBPs and microRNAs) in the ribonome. The ribonome feeds information to the proteome via translation and thereby shapes its composition, but it also has a reciprocal regulatory relationship with it that sustains and limits RBP production within the ribonome (double arrow). Horizontal RNA transfer to the ribonome to Cell \#2 (Valadi et al. 2007) via exosome-mediated exchange directly affects both its ribonome and its proteome through RNA delivery and translation, but thereby affecting the phenotype of the recipient cell (BajKrzyworzeka et al. 2006; Ratajczak et al. 2006; Aliotta et al. 2007.

necessarily encode RBPs. Thus, there is balanced control of multiple mRNAs that encode functionally related proteins, such as those that form macromolecular complexes and signaling pathways per the PTRO theory (Fig. 3) (Keene and Tenenbaum 2002). When a signal such as phosphorylation is directed at a specific RBP, it can affect the open system and at least temporarily override the regulatory effects of its neighboring RBPs in the ribonome (Intine et al. 2003 Benjamin et al. 2006; Abdelmohsen et al. 2007; Garbarino-Pico et al. 2007). Therefore, the "open" as well as the "closed" properties of the ribonome provide a dynamic quality of multilevel coordination that has not been appreciated by biologists in the past. Indeed, this theory posits that the ribonome is a central operating system of living organisms that communicates in several directions including feeding forward to the proteome, feeding back to the transcriptome, and feeding horizontally to other cells through exosomes or microvesicles (Fig. 5) (Keene 2007; Valadi et al. 2007). The cell-to-cell level of communication involves the horizontal transfer of genetic information as RNA. We propose that it is this power to transfer its self-sustaining, self-limiting regulatory information networks that gives the ribonome a central place in biology, making it vital to the origins and development of multicellular organisms.

\section{POSTTRANSCRIPTIONAL REGULATION OF BIOLOGICAL CLOCKS}

Biological clocks are logically among the most coordinated processes in living systems. From the mechanisms of circadian rhythms to the cell cycle, many studies have indicated examples of proteins and mRNAs that are posttranscriptionally regulated (Kim et al. 2002; Roenneberg and Merrow 2003; Majercak et al. 2004). In addition, some RBPs have been shown to undergo rhythmic changes during the 24-hour circadian period (Panda et al. 2002; Storch et al. 2002). For example, several laborato- ries have investigated changes in mRNA expression throughout the 24-hour circadian cycle in which the previously known proteins Period, Clock, Cry, and others were detected. Among the distilled data in these studies were cyclic changes in mRNAs that encode both transcription factors and RBPs. For example, transcription factors Zfp36 and Sox3 show circadian expression in mammalian organs, and the RNA regulators nocturnin (Ccr4 deadenylase) and butyrate response factor (BRF1) show cyclic circadian expression as well (Panda et al. 2002; Storch et al. 2002; Lidder et al. 2005). In addition, the RBP, LARK also cycles with circadian rhythms and was found to activate translation of the mRNA encoding the Period protein in the suprachiasmatic nucleus in the mouse brain (Kojima et al. 2007). Nocturnin, on the other hand, is a deadenylating enzyme that interacts with a subpopulation of mRNAs of yet unknown composition (Garbarino-Pico et al. 2007), whereas BRF1 specifically destabilizes mRNAs encoding immediate-early gene products under the control of a specific protein kinase (Stoecklin et al. 2002; Benjamin et al. 2006). With the demonstration that BRF1 cycles in a diurnal manner in peripheral organs, it is believed that it degrades immediate-early gene transcripts everyday. Moroni and coworkers (Benjamin et al. 2006) proposed that BRF1 functions as a circadian oscillating PTRO, thus offering an alternative or complementary mechanism of coordination in addition to transcription.

\section{CONCLUSIONS}

The proposed central role of the ribonome and the importance of RNA-protein and RNA-RNA interactions in regulating and, indeed, correlating outcomes of gene expression programs is based on evidence that such interactions are functionally related and contextually present in time and space in various cell types. How such interactions may affect biological clocks such as circadian 
rhythms in particular is unclear, but it is logical to predict that such coordinating principles like PTROs may have a role in oscillatory processes involving transcription and translation. However, the potential expansion of information transfer among surrounding cells in tissues and organs whether it involves PTROs per se presents a novel possibility for connections between biological clocks that depend on cycles in gene expression to work properly in both the central nervous system and the peripheral organs. Although evidence for such regulation has not been reported, it will be important to investigate this putative mode of information transfer as both a homeostatic and as an environmentally responsive system of intercellular communication and coordination.

\section{REFERENCES}

Abdelmohsen K., Pullmann R. Jr., Lal A., Kim H.H., Galban S., Yang X., Blethrow J.D., Walker M., Shubert J., Gillespie D.A., Furneaux H., and Gorospe M. 2007. Phosphorylation of HuR by Chk2 regulates SIRT1 expression. Mol. Cell 25: 543.

Aliotta J.M., Sanchez-Guijo F.M., Dooner G.J., Johnson K.W., Dooner M.S., Greer K.A., Greer D., Pimentel J., Kolankiewicz L.M., Puente N., Faradyan S., Ferland P., Bearer E.L., Passero M.A., Adedi M., Colvin G.A., and Quesenberry P.J. 2007. Alteration of marrow cell gene expression, protein production and engraftment into lung by lung-derived microvesicles: A novel mechanism for phenotype modulation. Stem Cells (in press). 25: 2245.

Antic D., Lu N., and Keene J.D. 1999. ELAV tumor antigen, Hel-N1, increases translation of neurofilament M mRNA and induces formation of neurites in human teratocarcinoma cells. Genes Dev. 13: 449.

Aten J.A. and Kanaar R. 2006. Chromosomal organization: Mingling with the neighbors. PLoS Biol. 4: e155.

Baj-Krzyworzeka M., Szatanek R., Weglarczyk K., Baran J., Urbanowicz B., Branski P., Ratajczak M.Z., and Zembala M. 2006. Tumour-derived microvesicles carry several surface determinants and mRNA of tumour cells and transfer some of these determinants to monocytes. Cancer Immunol. Immunother. 55: 808.

Bartel D. P. 2004. MicroRNAs: Genomics, biogenesis, mechanism, and function. Cell 116: 281.

Beckwith J. 1996. The operon: An historical account. In Escherichia coli and Salmonella cellular and molecular biology (ed. F.C. Neidhart), p. 1227. ASM Press, Washington, D.C.

Benjamin D., Schmidlin M., Min L., Gross B., and Moroni C. 2006. BRF1 protein turnover and mRNA decay activity are regulated by $\mathrm{PKB}$ at the same phosphorylation sites. Mol. Cell. Biol. 26: 9497.

Brennan C.M. and Steitz J.A. 2001. HuR and mRNA stability. Cell. Mol. Life Sci. 58: 266.

Brown V., Jin P., Ceman S., Darnell J. C., O’Donnell W. T., Tenenbaum S. A., Jin X., Feng Y., Wilkinson K. D., Keene J. D., Darnell R.B., and Warren S.T. 2001. Microarray identification of FMRP-associated brain mRNAs and altered mRNA translational profiles in fragile X syndrome. Cell 107: 477.

Eystathioy T., Chan E.K.L., Griffith K., Tenenbaum S.T., Keene J.D., and Fritzler M.J. 2002. A phosphorylated cytoplasmic autoantigen, GW182, associates with a unique population of human mRNAs within novel cytoplasmic speckles. Mol. Biol. Cell 13: 1338.

Fan J., Heller N.M., Gorospe M., Atasoy U., and Stellato C. 2005. The role of post-transcriptional regulation in chemokine gene expression in inflammation and allergy. Eur. Respir. J. 26: 933.

Gao F., Carson C., Levine T.D., and Keene J.D. 1994. Selection of a subset of mRNAs from 3'UTR combinatorial libraries using neuronal RNA-binding protein, Hel-N1. Proc. Natl.
Acad. Sci. 91: 11207.

Garbarino-Pico E., Niu S., Rollag M.D., Strayer C.A., Besharse J.C., and Green C.B. 2007. Immediate early response of the circadian polyA ribonuclease nocturnin to two extracellular stimuli. $R N A$ 13: 745 .

Gerber A.P., Herschlag D., and Brown P.O. 2004. Extensive association of functionally and cytotopically related mRNAs with Puf family RNA-binding proteins in yeast. PLoS Biol. 2: e79.

Gerber A.P., Luschnig S., Krasnow MA, Brown P.O., and Herschlag D. 2006. Genome-wide identification of mRNAs associated with the translational regulator PUMILIO in Drosophila melanogaster. Proc. Natl. Acad. Sci. 103: 4487.

Grimson A., Farh K.K.-H., Johnston W.K., Garrett-Engele P., Lim L.P., and Bartel D.P. 2007. MicroRNA targeting specificity in mammals: Determinants beyond seed pairing. Cell 27: 91 .

Hieronymus H. and Silver P.A. 2003. Genome-wide analysis of RNA-protein interactions illustrates specificity of the mRNA export machinery. Nat. Genet. 33: 155 .

. 2004. A systems view of mRNP biology. Genes Dev. 18: 2845.

Intine R.V., Tenenbaum S.A., Sakulich A.L., Keene J.D., and Maraia R.J. 2003. Differential phosphorylation and subcellular localization of La RNPs associated with precursor tRNAs and translation-related mRNAs. Mol. Cell 12: 1301.

Jain R.G., Andrews L.G., McGowan K.M., Pekala P., and Keene J.D. 1997. Ectopic expression of Hel-N1, an RNA-binding protein, increases glucose transporter (GLUT1) expression in 3T3-L1 adipocytes. Mol. Cell. Biol. 17: 954.

Keene J.D. 1999. Why is Hu where? Shuttling of early-responsegene messenger RNA subsets. Proc. Natl. Acad. Sci. 96: 5. . 2001. Ribonucleoprotein infrastructure regulating the flow of genetic information between the genome and the proteome. Proc. Natl. Acad. Sci. 98: 7018.

2003. Organizing mRNA export. Nat. Genet. 33: 111.

2007. RNA regulons: Coordination of posttranscriptional events. Nat. Rev. Genet. 8: 533.

Keene J.D. and Lager P.J. 2005. Post-transcriptional operons and regulons co-ordinating gene expression. Chromosome Res. 13: 327.

Keene J.D. and Tenenbaum S.A. 2002 Eukaryotic mRNPs may represent posttranscriptional operons. Mol. Cell 9: 1161.

Keene J.D., Komisarow J.M., and Friedersdorf M.B. 2006. RIPChip: The isolation and identification of mRNAs, microRNAs and protein components of ribonucleoprotein complexes from cell extracts. Nat. Protoc. 1: 1.

Kim E.Y., Bae K., Ng F.S., Glossop N.R.J., Hardin P.E., and Edery I. 2002. Drosophila CLOCK protein is under posttranscriptional control and influences light-induced activity. Neuron 34: 69.

King P.H., Levine T.D., Fremeau R.T., and Keene J.D. 1994. Mammalian homologs of Drosophila ELAV localized to a neuronal subset can bind in vitro to the 3' UTR of mRNA encoding the Id transcriptional repressor. J. Neurosci. 14: 1943.

Kojima S., Matsumoto K., Hirose M., Shimada M., Nagano M., Shigeyoshi Y., Hoshino S., Ui-Tei K., Saigo K., Green C.B., Sakaki Y., and Tei H. 2007. LARK activates posttranscriptional expression of an essential mammalian clock protein, PERIOD1. Proc. Natl Acad. Sci. 104: 1859.

Kosak S.T. and Groudine M. 2004. Form follows function: The genomic organization of cellular differentiation. Genes Dev. 18: 1371.

Lercher M.J., Chamary J.V., and Hurst L.D. 2004. Genomic regionality in rates of evolution is not explained by clustering of genes of comparable expression profile. Genome Res. 14: 1002.

Levine T.D., Gao F., King P.H., Andrews L.G., and Keene J.D. 1993. Hel-N1: An autoimmune RNA-binding protein with specificity for 3' uridylate-rich untranslated regions of growth factor mRNAs. Mol. Cell. Biol. 13: 3494.

Lewis B.P., Burge C.B., and Bartel D.P. 2005. Conserved seed pairing, often flanked by adenosines, indicates that thousands of human genes are microRNA targets. Cell 120: 15 . 


\section{RNA OPERONS, REGULONS, AND BIOLOGICAL CLOCKS}

Lewis B.P., Shih I.H., Jones-Rhoades M.W., Bartel D.P., and Burge C.B. 2003. Prediction of mammalian micro RNA targets. Cell 115: 787.

Lidder P., Gutierrez R.A., Salome P.A., McClung C.R., and Green P.J. 2005. Circadian control of messenger RNA stability. Association with a sequence-specific messenger RNA decay pathway. Plant Physiol. 138: 2374.

Lopez de Silanes I., Zhan M., Lal A., Yang X., and Gorospe M. 2004. Identification of a target RNA motif for RNA-binding protein HuR. Proc. Natl. Acad. Sci. 101: 2987.

Ma W.J., Chang S., Campbell C., Wright A., and Furneaux H. 1996. Cloning and characterization of HuR, a ubiquitously expressed Elav-like protein. J. Biol. Chem. 271: 8144.

Maniatis T. and Reed R. 2002. An extensive network of coupling among gene expression machines. Nature 416: 499.

Majercak J., Chen W.-F., and Edery I. 2004. Splicing of period $3^{\prime}$ terminal intron is regulated by light, circadian clock factors and phospholipase C. Mol. Cell. Biol. 24: 3359.

Mazan-Mamczarz K., Galban S., Lopez de Silanes I., Martindale J.L., Atasoy U., Keene J.D., and Gorospe M. 2003. RNAbinding protein $\mathrm{HuR}$ enhances p53 translation after ultraviolet light irradiation. Proc. Natl. Acad. Sci. 100: 8354.

Moore M.J. 2005. From birth to death: The complex lives of eukaryotic mRNAs. Science 309: 1514.

Niehrs C. and Pollet N. 1999. Synexpression groups in eukaryotes. Nature 402: 483.

Orphanides G. and Reinberg D. 2002. A unified theory of gene expression. Cell 108: 439.

Panda S., Antoch M.P., Miller B.H., Su A.I., Schook A.B., Straume M., Schultz P.G., Kay S.A., Takahashi J.S., and Hogenesch J.B. 2002. Coordinated transcription of key pathways in the mouse by the circadian clock. Cell 109: 307.

Penalva L.O., Tenenbaum S.A., and Keene J.D. 2004. Gene expression analysis of messenger RNP complexes. Methods Mol. Biol. 257: 125.

Ratajczak J., Miekus K., Kucia M., Zhang J., Reca R., Dvorak P., and Ratajczak M.Z. 2006. Embryonic stem cell-derived microvesicles reprogram hematopoietic progenitors: Evidence for horizontal transfer of mRNA and protein delivery. Leukemia 20: 847

Richter J.D. 1997. mRNA formation and function. Academic Press, New York.

Robinow S., Campo A.R., Ya K.M., and White K. 1988. The elav gene product of Drosophila, required in neurons, has three RNP consensus motifs. Science 242: 1570

Rodriguez-Trelles F., Tarrio R., and Ayala F.J. 2005. Is ectopic expression caused by deregulatory mutations or due to gene-regulation leaks with evolutionary potential? Bioessays 27: 592.

Roenneberg T. and Merrow M. 2003. The network of time:
Understanding the molecular circadian system. Curr. Biol. 13: R198.

Shaw G. and Kamen R. 1986. A conserved AU sequence from the 3' untranslated region of GM-CSF mRNA mediates selective mRNA degradation. Cell 46: 659 .

Spellman P.T. and Rubin G.M. 2002. Evidence for large domains of similarly expressed genes in the Drosophila genome. J. Biol. 1: 5.

Stoecklin G., Colombi M., Raineri I., Leuenberger S., Mallaun M., Schmidlin M., Gross B., Lu M., Kitamura T., and Moroni C. 2002. Functional cloning of BRF1, a regulator of AREdependent mRNA turnover. EMBO J. 21: 4709.

Storch K.F., Lipan O., Leykin I., Viswanathan N., Davis F.C., Wong W.H., and Weitz C.J. 2002. Extensive and divergent circadian gene expression in liver and heart. Nature 417: 78.

Szabo A., Dalmau J., Manley G., Rosenfeld M., Wong E., Henson J., Posner J.B., and Furneaux H.M. 1991. HuD, a paraneoplastic encephalomyelitis antigen, contains RNABinding domains and is homologous to Elav and Sex-lethal. Cell 67: 325.

Tenenbaum S.A., Carson C.C., Atasoy U., and Keene J.D. 2003. Genome-wide regulatory analysis combining en masse nuclear run-ons (emRUNs) and ribonomic profiling. Gene 317: 79 .

Tenenbaum S.A., Carson C.C., Lager P.J. and Keene J.D. 2000. Identifying mRNA subsets in messenger ribonucleoprotein complexes by using cDNA arrays. Proc. Natl. Acad. Sci. 97: 14085.

Tenenbaum S.A., Lager P.J., Carson C.C., and Keene J.D. 2002. Ribonomics: Identifying mRNA subsets in mRNP complexes using antibodies to RNA-binding proteins and genomic arrays. Methods 26: 191.

Townley-Tilson W.H., Pendergrass S.A., Marzluff W.F., and Whitfield M.L. 2006. Genome-wide analysis of mRNAs bound to the histone stem-loop binding protein. RNA 12: 1853.

Valadi H., Ekström K., Bossios A., Sjöstrand M., Lee J.J., and Lötvall J.O. 2007. Exosome-mediated transfer of mRNAs and microRNAs is a novel mechanism of genetic exchange between cells. Nat. Cell Biol. 9: 654 .

Vemuri G.N. and Aristidou A.A. 2005. Metabolic engineering in the -omics era: Elucidating and modulating regulatory networks. Microbiol. Mol. Biol. Rev. 69: 197.

Wilusz C.J. and Wilusz J. 2004. Bringing the role of mRNA decay in the control of gene expression into focus. Trends Genet. 20: 491.

Yanai I., Korbel J.O., Boue S., McWeeney S.K., Bork P., and Lercher M.J. 2006. Similar gene expression profiles do not imply similar tissue functions. Trends Genet. 22: 132. 


\section{$\overbrace{\text { CSH' }}^{\infty}$ Cold Spring Harbor Symposia SYMPOSIA}

\section{Biological Clocks and the Coordination Theory of RNA Operons and Regulons}

J. D. Keene

Cold Spring Harb Symp Quant Biol 2007 72: 157-165

Access the most recent version at doi:10.1101/sqb.2007.72.013

References This article cites 63 articles, 27 of which can be accessed free at:

http://symposium.cshlp.org/content/72/157.full.html\#ref-list-1

License

Email Alerting Receive free email alerts when new articles cite this article - sign up in the box at the Service top right corner of the article or click here.

To subscribe to Cold Spring Harbor Symposia on Quantitative Biology go to: http://symposium.cshlp.org/subscriptions 